\title{
Exploring the temporal dynamics of social facilitation in the Stroop task
}

\author{
Dinkar Sharma ANd Rob Booth \\ University of Kent, Canterbury, England \\ RUPERT BROWN \\ University of Sussex, Brighton, England \\ AND \\ Pascal Huguet \\ CNRS and Université Aix-Marseille, Aix-en-Provence, France
}

\begin{abstract}
The importance of social context in affecting attention has recently been highlighted by the finding that the presence of a passive, nonevaluative confederate can improve selective attention. The underlying mechanism, however, remains unclear. In this paper, we argue that social facilitation can be caused by distractor inhibition. Two distinct sources of evidence are provided from an experiment employing the Stroop task with and without social presence. First, analysis of the response time (RT) distribution indicates that interference is reduced at relatively long RTs. This is consistent with an inhibitory mechanism, whose effects build up slowly. Further support is provided by showing that social facilitation is prevented by using short response-to-stimulus intervals that are thought to reduce cognitive control processes.
\end{abstract}

Cognitive control involves resolving the competition between competing processes; for example, word reading and color naming compete in a Stroop (1935) task. The dominant tendency to read the word must be overcome in favor of responding to the color. Stroop interference occurs when responses are slower to incongruent colorword stimuli (e.g., the word RED in blue print) than to nonlexical stimuli (e.g., +++ in blue print; for a review, see MacLeod, 1991). Stroop interference can be affected by informational or cognitive contexts (e.g., Melara \& Algom, 2003). In this article, we show how social contexts can affect Stroop interference.

Previous research has shown that Stroop interference is reduced by the presence of a passive, nonevaluative observer (Huguet, Galvaing, Monteil, \& Dumas, 1999). This is important, because it helps resolve a long-standing controversy about the cause of social facilitation effects. Huguet et al. favored distraction-conflict theory (DCT; Baron, 1986), which states that the presence of an observer leads to "attentional conflict": the participant must attend to both the task and the observer. Such attentional conflict threatens the participant with cognitive overload, leading to a restriction in attention focus (e.g., Cohen, 1978). This can aid or hinder performance, depending on the task in question. In the Stroop task, the presence of an observer should make participants less likely to attend to the irrelevant word. This leads to the prediction that social presence reduces Stroop interference, as found by Huguet et al. (1999; for similar effects in the real or imaginary presence of a coactor, see Dumas, Huguet, \& Ayme, 2005; Huguet, Dumas, \& Monteil, 2004).

However, there are some problems with this account. First, DCT predicts that audience presence leads to the use of a restricted range of cues. Although some research supports this notion (Muller, Atezeni, \& Butera, 2004), other research suggests this would not reduce Stroop interference. For example, Chen (2003) found that narrowing attentional focus increased, rather than decreased, interference. Second, DCT is an "early selection" account, in that it assumes that social presence reduces the likelihood of processing the distractor. Consistent with this idea, Huguet et al. (1999) found that recognition memory for the distractor words was reduced in the presence condition, as one would expect, if word meaning was filtered out. However, this was not replicated in more recent experiments, despite reduced Stroop interference being found (see Dumas et al., 2005; Huguet et al., 2004). This pattern of reduced Stroop interference with intact recognition suggests a "late selection" account of social facilitation in the Stroop task: The distractor is processed as normal, but is then strongly inhibited before a response is selected.

\section{Distractor Inhibition}

It is commonly accepted that inhibition of undesirable stimuli is important for attentional selection. One impor-

D.Sharma, d.sharma@kent.ac.uk 
tant feature of inhibition is that it builds relatively slowly, peaking some time after stimulus onset (see Eimer, 1999; Eimer \& Schlaghecken, 1998; Houghton \& Tipper, 1994; Ridderinkhof, van den Wildenberg, Wijnen, \& Burle, 2004). This buildup of inhibition is central to our account of social facilitation, so this experiment aimed to measure it directly, using a method developed by Ridderinkhof and colleagues (Ridderinkhof, 2002; Ridderinkhof, Scheres, Oosterlaan, \& Sergeant, 2005; Ridderinkhof et al., 2004). Any Stroop experiment yields two response-time (RT) distributions, one for incongruent trials, and one for control trials (Figure 1A). The horizontal (RT) difference between the two distributions represents the amount of Stroop interference. This difference can be plotted against overall RT, producing a delta plot (Figure 1B), which shows how interference develops with time in the "average" trial.

Typically, interference increases monotonically with RT, since the two distributions are not tied to each other and the incongruent distribution has a longer tail. Inhibition can decrease interference, but because inhibition is reactive and builds slowly, its effects will be stronger for longer RTs. Inhibition will therefore tend to flatten the later part of the delta plot. Where interference decreases at longer RTs, this is diagnostic of strong inhibition (squares in Figure 1). This is the first time in social facilitation research that an experiment has looked for such a pattern in social presence.

The effects of cognitive control on task performance also depend on the interval between successive stimuli. In our study, participants completed two blocks of Stroop trials, one with the 1,000-msec response-stimulus interval (RSI) typical of social facilitation Stroop experiments, and

A

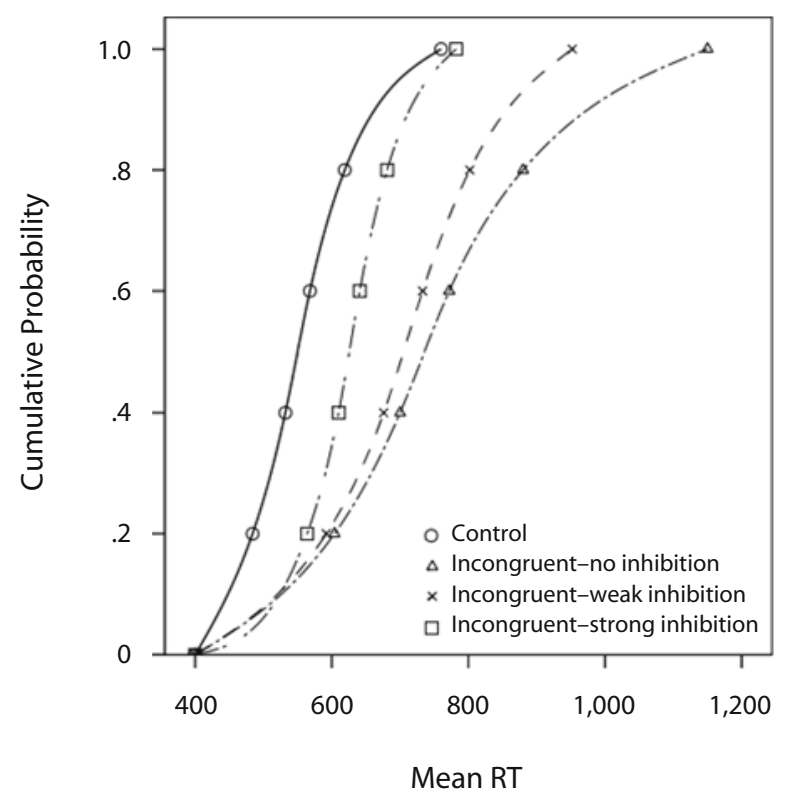

one with a 32-msec RSI. RTs increase as RSI decreases (Rabbitt \& Maylor, 1991); one suggestion is that reducing RSI prevents adequate preparation for each trial (Rabbitt, 1980; Sharma \& McKenna, 2001). If social facilitation is also dependent on cognitive control processes that occur in the interval between successive trials, using an RSI of $32 \mathrm{msec}$ should reduce their influence. In contrast, early selection processes, such as those described in DCT, are less affected by cognitive control and so should be unaffected by manipulations of RSI.

\section{Mere Effort}

Our late selection approach comes close to Harkins's (2006; Jamieson \& Harkins, 2007) "mere effort" account of social facilitation. This states that the potential for evaluation that accompanies social presence motivates the participants to do well, which potentiates their dominant response tendency. This would increase Stroop interference. However, when the dominant response is easily identified as incorrect, participants under evaluation are especially motivated to inhibit it; and this requires time and cognitive resources.

Consistent with these arguments, McFall, Jamieson, and Harkins (2009) found that experimenter evaluation only reduced Stroop interference when the response deadline was late enough $(2 \mathrm{sec})$ for cognitive inhibition to operate (as in Huguet et al., 1999, where there was no deadline). Faced with a shorter deadline (750 msec or $1 \mathrm{sec}$ ), participants showed no difference in RT interference when subject to evaluation, but made more errors on incongruent trials. ${ }^{1}$ Thus, the mere-effort account and our late-selection approach both assume that social fa-

Figure 1. Cumulative probability functions for response time (A), and delta plot (B), for incongruent and control conditions in a hypothetical Stroop experiment. Delta plots illustrate the impact of no, weak, and strong inhibition on interference (incongruent control). Figure adapted from Ridderinkhof et al. (2005, Figures A3.A and A3.B). 
cilitation of Stroop performance is rooted in the late inhibition of word information. However, research so far has neglected the RT distribution, which is crucial to the inhibition argument.

\section{METHOD}

\section{Participants}

Forty-eight university students (24 males) were paid $£ 2$ for participation.

\section{Design and Materials}

The experiment employed a $2 \times 2 \times 2$ mixed design with two within-participants factors - trial type (incongruent color word, control) and RSI (32 msec, 1,000 msec) — and one between-participants factor-social context (alone, presence).

Stimuli comprised the incongruent color words RED, BLUE, YELLOW, and GREEN, and control strings of "+ "s of equal length to these words, appearing once in each of the other three colors and repeated three times each, to form 72 randomly ordered trials for each RSI condition. A practice phase of 128 trials comprised the words TABLE, CHAIR, RUG, LAMP, DESK, MIRROR, SHELF, and SOFA, presented four times in each color. Stimuli were presented centrally against a black background using a computer (Dell Inspiron 510M) running E-Prime 1.1.4.1. Responses were made using a PST response box, with four buttons labeled with the four target colors. Participants were tested in a laboratory with a seat placed directly to the right of the screen, so that the confederate was clearly visible in the "presence" condition.

\section{Procedure}

Participants were recruited by an experimenter of the opposite gender. The Stroop task was described, and the requirement to respond to the colors as quickly and accurately as possible, while ignoring the words, was emphasized. The experimenter said that they were conducting another study in the adjacent room and that he/she had to wait outside for another participant. The participants were instructed to call the experimenter when they had completed the practice trials. The RSI was always $32 \mathrm{msec}$ during practice.

The experimental phase consisted of two blocks with a short break. The blocks were identical, except that one employed an RSI of $32 \mathrm{msec}$ and the other an RSI of 1,000 msec, with order counterbalanced across participants.

In the "presence" condition, the experimenter said that another participant had arrived for the adjacent experiment but that the computer had developed a fault; the experimenter then asked if the confederate could sit in the room while this was fixed, explaining that the other experiment presented auditory stimuli to which the confederate could not be exposed. None refused to allow this. The confederate sat in the only available seat, next to the screen. The experimenter said that the participant was completing a Stroop task, and asked if the confederate knew anything about the task: the confederate replied in the negative. The confederate was always of the same gender as the participant. During the experimental trials, the confederate looked at the participants' faces and hands during 40 trials from each block, and read a book for the remaining 32 . This replicates Huguet et al.'s (1999) procedure, in which participants were observed $60 \%-70 \%$ of the time. The experimenter left the room during the task in both the alone and presence conditions. ${ }^{2}$

\section{RESULTS}

\section{RTs}

Correct RTs (95.43\% of the data) were subjected to a nonrecursive outlier-trimming procedure (removing a further $2.41 \%$ ) as described by Van Selst and Jolicœur
(1994). The data of 1 participant, who misunderstood the instructions, were excluded. Mean RTs were subjected to a $2 \times 2 \times 2 \times 2$ mixed ANOVA, with trial type, RSI, social context, and order of the RSI blocks as factors.

Not surprisingly, there was a significant Stroop effect: incongruent $=774 \mathrm{msec}$, control $=709 \mathrm{msec}[F(1,42)=$ 36.56, $\left.M S_{\mathrm{e}}=5,331, p<.001, \eta_{\mathrm{p}}^{2}=.465\right]$. Likewise, a main effect of RSI was also found $[F(1,42)=52.32$, $\left.M S_{\mathrm{e}}=5977, p<.001, \eta_{\mathrm{p}}^{2}=.555\right]$; as expected, responses were quicker in the long-RSI condition $(M=700)$ than in the short-RSI condition $(M=783)$. Trial type did not interact with context $\left[F(1,42)=0.84, M S_{\mathrm{e}}=5,331, p=\right.$ $\left..36, \eta_{\mathrm{p}}^{2}=.020\right]$ or RSI $\left[F(1,42)=0.44, M S_{\mathrm{e}}=1,928\right.$, $\left.p=.51, \eta_{\mathrm{p}}^{2}=.010\right]$. There was no main effect of social context $\left[F(1,42)=0.51, M S_{\mathrm{e}}=98,686, p=.48, \eta_{\mathrm{p}}^{2}=\right.$ $0.01]$; however, context did interact with RSI $[F(1,42)=$ 5.91, $\left.M S_{\mathrm{e}}=5,977, p<.05, \eta_{\mathrm{p}}^{2}=.123\right]$. Simple main effects analysis indicated that social context did not affect RTs in either RSI condition (both $F \mathrm{~s}<1.5, p \mathrm{~s}>.2$ ), but the simple main effect of RSI was larger in the alone condition $[F(1,20)=34.5, p<.001]$ than in the presence condition $[F(1,22)=16.4, p<.01]$.

More importantly, the predicted interaction between trial type, RSI, and context was significant $[F(1,42)=$ 4.91, $\left.M S_{\mathrm{e}}=1,928, p<.05, \eta_{\mathrm{p}}^{2}=.105\right]$ (see Figure 2). As predicted, the presence of the confederate reduced Stroop interference in the long-RSI condition $[F(1,42)=4.29$, $\left.M S_{\mathrm{e}}=3,150, p<.05, \eta_{\mathrm{p}}^{2}=.093\right]$, but not in the short-RSI condition $\left[F(1,42)=0.11, M S_{\mathrm{e}}=4,109, p=.74, \eta_{\mathrm{p}}^{2}=\right.$ .003]. Simple main effect of presence was not significant for the control condition under long or short RSI (both $\left.F_{\mathrm{s}}<2.23, p>.14\right)$.

There was no main effect of order, and no interaction involving order with social context (all $F_{\mathrm{s}}<2.48$, $p \mathrm{~s}>.1)$. However, order did interact with RSI and trial type $\left[F(1,42)=22.36, M S_{\mathrm{e}}=1,928, p<.01, \eta_{\mathrm{p}}^{2}=.347\right]$. This indicated that Stroop interference was reduced with practice (when presented first, short $=103 \mathrm{msec}$, long = $90 \mathrm{msec}$; when presented second, short $=10 \mathrm{msec}$, long = $50 \mathrm{msec})$.

\section{Error Rates}

Errors were subjected to the same analyses. There was a significant Stroop effect $(M=.040$ for incongruent trials, .032 for control trials) $\left[F(1,42)=4.06, M S_{\mathrm{e}}=0.001, p=\right.$ $\left..05, \eta_{\mathrm{p}}^{2}=.088\right]$; no other effects were significant (all $F \mathrm{~s}<$ 2.55 , all $p \mathrm{~s}>.11)$.

\section{Time Course Analyses}

RT distributions were examined for evidence of distractor inhibition, as described by Ridderinkhof et al. (2005; Ridderinkhof et al., 2004). RTs were divided by participant and condition, and rank ordered. RTs over $3,000 \mathrm{msec}$ were excluded as errors; this did not greatly affect the pattern of results, however. Remaining observations were divided into four "bins" of nine RTs per condition per participant. The mean RT for each incongruent bin was then compared with that for the corresponding control bin to yield a "delta" or interference score (see Figure 3). 
Long RSI

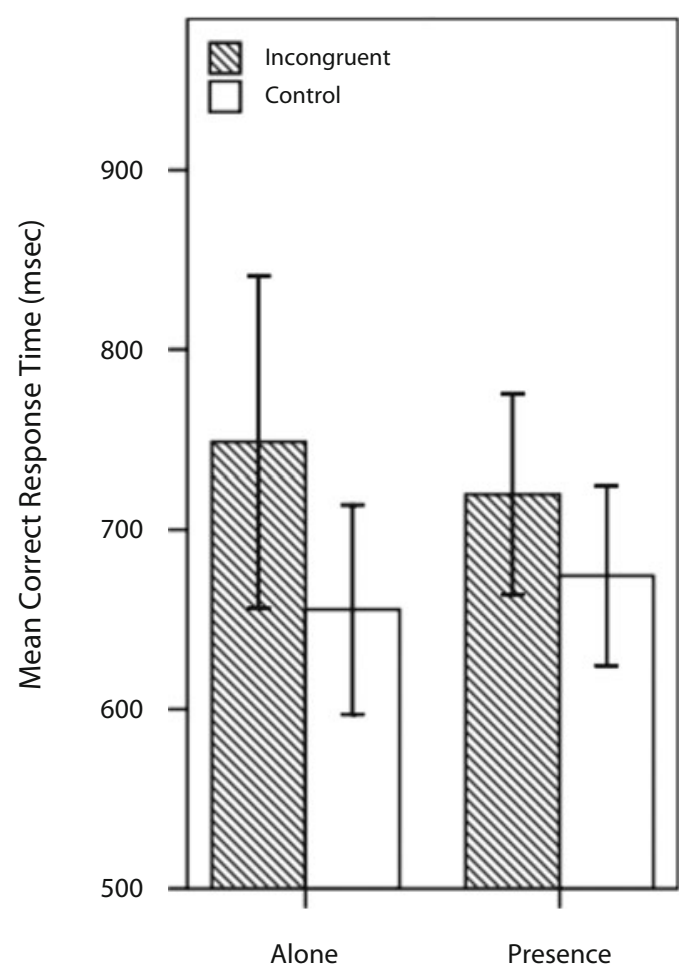

Short RSI

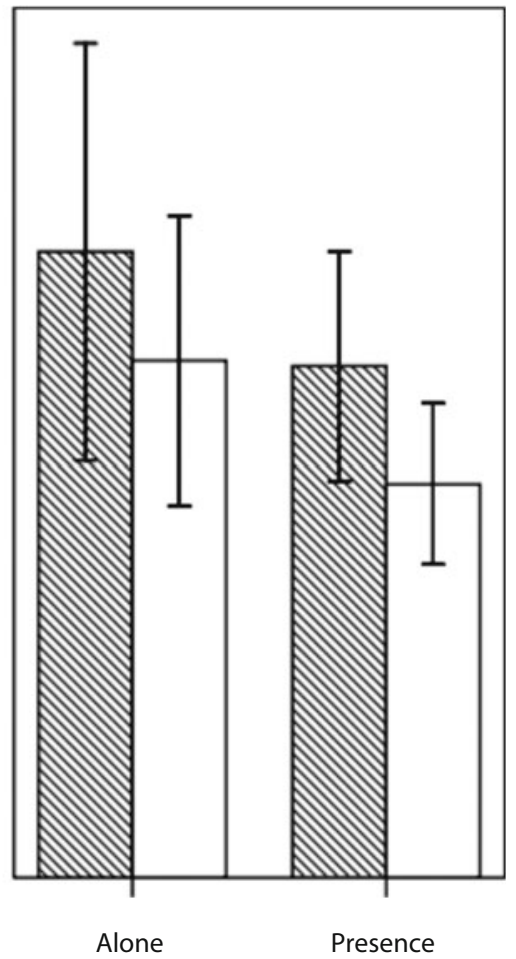

Social Context

Figure 2. Overall performance in the Stroop task. Error bars are \pm 1 standard error.

The critical variable when analyzing delta plots is the point at which the two lines, here the alone and presence conditions, diverge. To this end, mean gradients between the bins were calculated. Planned comparisons on the long-RSI condition revealed a marginal difference between the alone and presence conditions for the period between Bins 1 and $2[t(44)=1.98, p=.054]$, no difference between Bins 2 and $3[t(44)=1.11, p=.28]$, and a significant difference between Bins 3 and $4[t(44)=2.96$, $p<.01]$. This indicates that the alone and presence conditions had fully diverged by the end of the RT distribution. Planned comparisons on the short-RSI condition revealed that the alone and presence conditions did not diverge (all $t \mathrm{~s}<1.1, p \mathrm{~s}>.3$ ). In summary, these plots show that interference increases with RT in the short-RSI (both alone and presence) and long-RSI/alone conditions. However, in the long-RSI/presence condition, the interference never rises: It is flat and then falls.

\section{DISCUSSION}

This study replicates previous work showing that the presence of others reduces Stroop interference with a 1,000-msec RSI (Huguet et al., 1999), but in addition shows that this reduction does not occur, as expected, with a 32-msec RSI. It seems that short RSIs can prevent efficient attentional control, perhaps by preventing the participant from adequately preparing for each trial. This is consistent with an inhibition account of social facilitation.

The delta plots also suggest that social facilitation in the long RSI condition occurred because of distractor inhibition: Ridderinkhof et al. (2005) stated that a decreasing plot provides the strongest evidence of inhibition. These new data suggest that the distractors are not deselected and left unprocessed, as suggested by DCT, but provide the first strong evidence for a later, reactive process.

The present findings also partially support Harkins's (2006) "mere effort" account of social facilitation. According to this account, evaluation motivates the participant and potentiates the prepotent response, but this is corrected when the response deadline is long enough to make a correction (leading to facilitation in the Stroop task; McFall et al., 2009). Here there was no significant decrease in RT in the presence condition, which contradicts a motivational account. Our study also had no response deadline, which, according to Harkins, should allow distractor inhibition to occur in motivated (observed) participants. The delta plots indeed show strong inhibition, in the long RSI/presence condition. No inhibition was found, however, in the short RSI/presence condition. This indicates that a late response deadline is not sufficient for distractor inhibition/correction to occur. As suggested above, it seems that social facilitation is also dependent on cogni- 


\section{Long RSI}
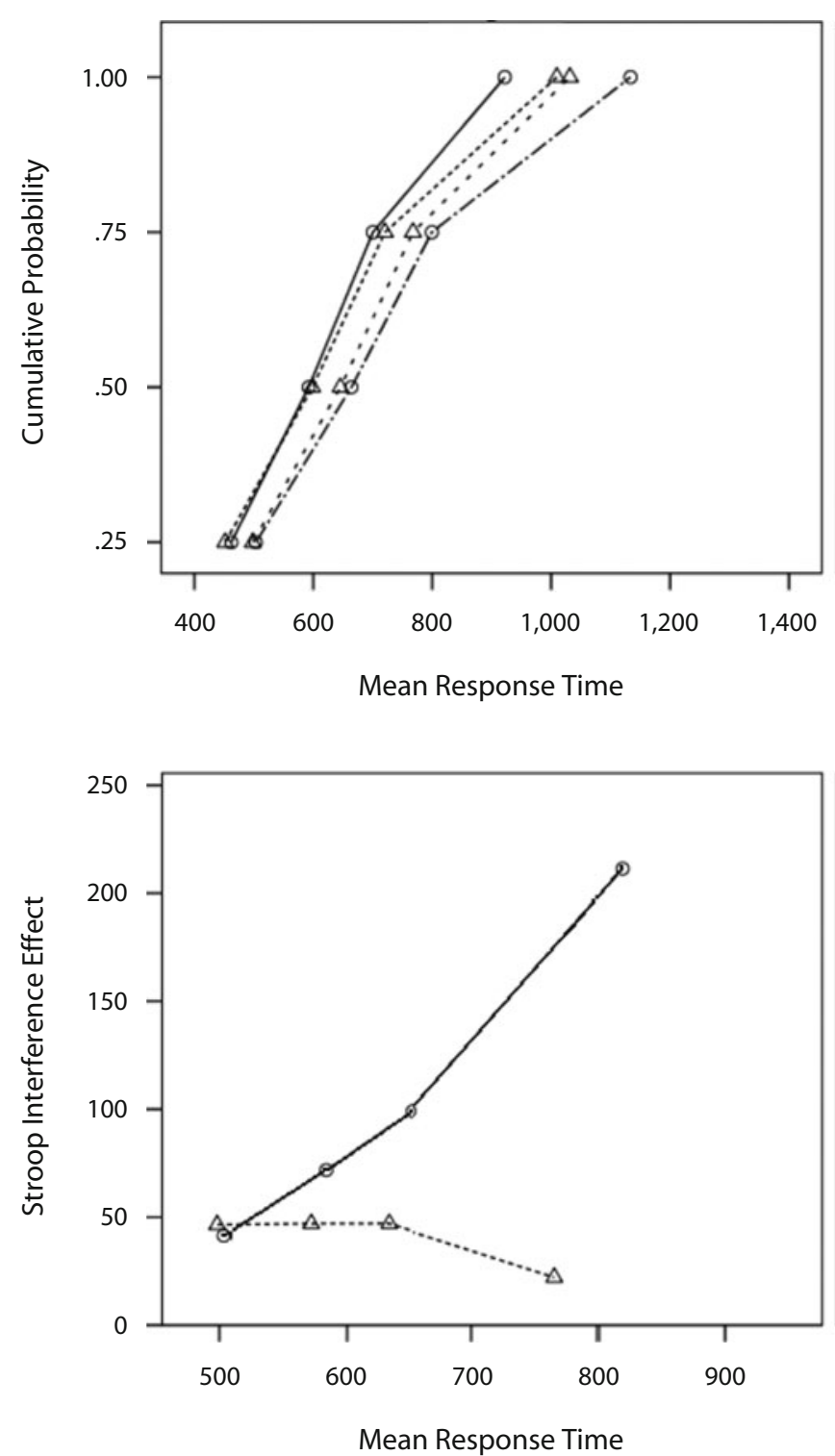

Short RSI

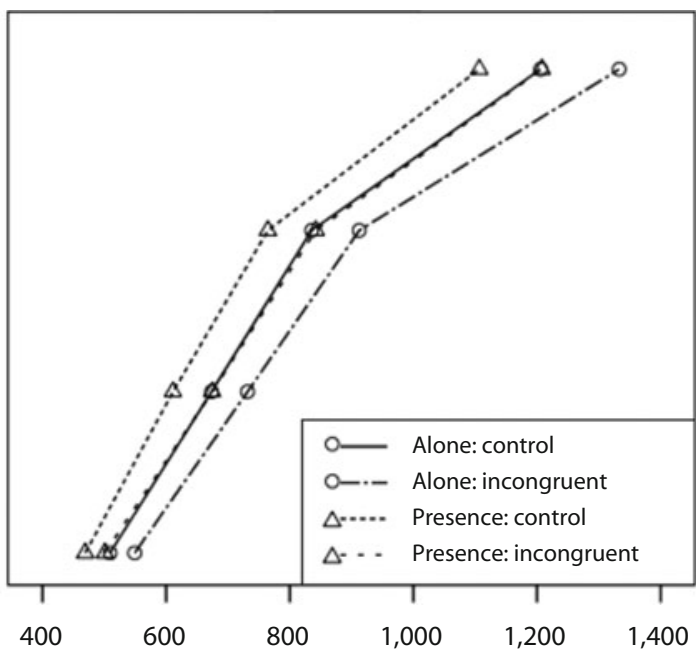

Mean Response Time

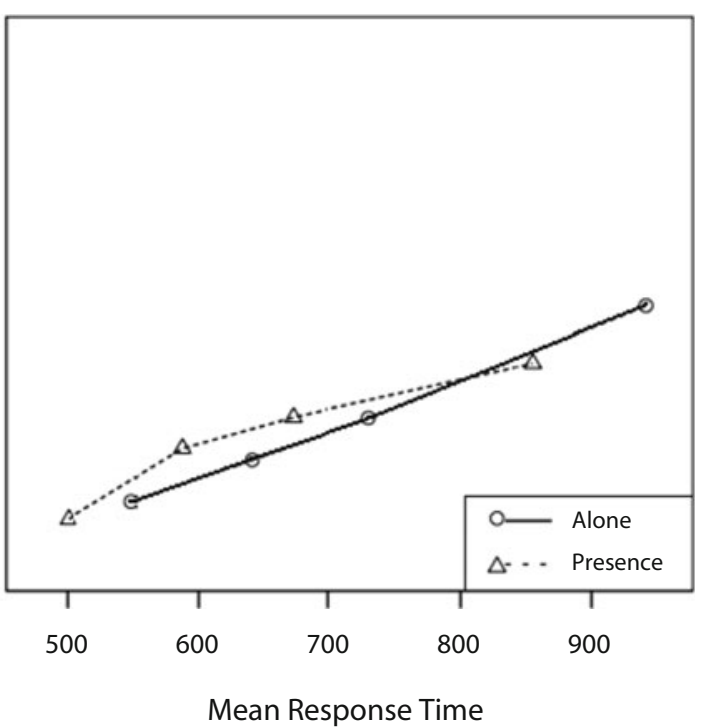

Figure 3. Cumulative probability functions (above) and delta plots (below) showing the time course of Stroop interference effects. Response times and interference effects are given in milliseconds.

tive control processes that occur in the interval between successive trials, such as preparation. Thus, the present results offer the first evidence that inhibition can be affected online by the presence of other people, provided there is sufficient time to prepare for each trial. ${ }^{3}$ This is the key contribution of the present article.

Another contribution merits attention. Klauer, Herfordt, and Voss (2008) noted that the Stroop interference in Huguet et al.'s (1999) experiment was unusually large in the alone condition, and argued that Huguet et al.'s social facilitation effects were in fact an artifact of this. To minimize evaluation apprehension, Huguet et al.'s (1999) participants were told that the computer was not yet programmed to record their responses, and that the goal of the session was to give their general impression about this new task. Klauer et al. hypothesized that this introduced a second task (impression formation) in addition to the Stroop task itself. Participants performing alone were more likely to spend time inspecting the more interesting incongruent trials, leading to very high interference. Klauer et al. replicated unusually large Stroop interference in the alone/impression formation-instruction condition. However, social presence still tended to be associated with reduced Stroop interference when the dual task instruction was removed, at least when participants performed alone first, with the presence condition second. ${ }^{4}$

The present research refutes Klauer et al.'s (2008) position. Here, the dual task instructions were removed, but 
the social facilitation remained, at least under long RSI, as expected. However, it remains possible that the present findings result from participants' spending more time on incongruent trials when they are not under time pressure (i.e., the long-RSI condition) and are less motivated to perform quickly (when they are not being observed). Neither the alone/long-RSI condition nor the presence/long-RSI condition differed from either of the short-RSI conditions in terms of overall interference ( $t \mathrm{~s}<1.4, p \mathrm{~s}>.17)$, but the time course analyses are arguably more revealing: When overall mean gradients were calculated for the four delta plots in Figure 3, planned comparisons revealed that the alone/long-RSI plot was steeper than the presence/longRSI plot $[t(44)=3.22, p<.01]$, and tended to be steeper than the alone/short-RSI plot $[t(21)=1.78, p=.09]$ and the presence/short-RSI plot $[t(44)=1.96, p=.056]$. The presence/long-RSI plot did not differ from either short-RSI plot $(t \mathrm{~s}<1.4, p \mathrm{~s}>.18)$. These results may suggest that, for long RSIs, participants are indeed inhibiting less than usual in the alone condition, rather than inhibiting more than usual in the presence condition. However, this assumes that participants respond more quickly in the presence of the confederate, and this effect was not found. Furthermore, it is unclear whether the absolute gradient of the delta plot is interpretable to the same degree as its shape (Ridderinkhof et al., 2005). There is a qualitative difference in the shape of the alone/long-RSI plot compared with the other three, in that it never rises, and actually decreases at longer RTs. This is impossible to account for in terms of Klauer et al.'s account. Further research should differentiate between Klauer et al.'s account of social facilitation and ours, perhaps by increasing the interest value of control stimuli.

Finally, future research should clarify whether the supposedly anodyne presence of the experimenter also makes a difference regarding distractor inhibition. This is an important question for experimental psychology in general, as there is always an experimenter present at some points in time during the experimental session. McFall et al.'s (2009) findings show that participants' performance can be affected by their beliefs as to how interested the experimenter is on individual performance. Another important question is whether the experimenter's physical presence can also make a difference: earlier social facilitation findings suggest it does (for a review, see Guerin, 1993). Given the present findings, this presence should be influential even when participants believe the experimenter's focus is on collective performance, a thought that merits special attention in future research.

\section{AUTHOR NOTE}

This research was supported by ESRC Grant RES-000-22-0534 to Dinkar Sharma and Rupert Brown. We thank Kristina Massey for help with data collection. Correspondence concerning this article should be addressed to D. Sharma, School of Psychology, University of Kent, Canterbury, Kent, CT2 7NP, England (e-mail: d.sharma@kent.ac.uk).

\section{REFERENCES}

Baron, R. S. (1986). Distraction-conflict theory: Progress and problems. In L. Berkowitz (Ed.), Advances in experimental social psychology (Vol. 19, pp. 1-36). Orlando: Academic Press.
CHEn, Z. (2003). Attentional focus, processing load, and Stroop interference. Perception \& Psychophysics, 65, 888-900.

CoHEN, S. (1978). Environmental load and the allocation of attention. In A. Baum, J. E. Singer, \& S. Valins (Eds.), Advances in environmental psychology (pp. 1-30). Hillsdale, NJ: Erlbaum.

Dumas, F. Huguet, P. \& Ayme, E. (2005). Social context effects in the Stroop task: When knowledge of one's relative standing makes a difference. Current Psychology Letters: Cognition, Brain, \& Behaviour, 16, 1-12.

EIMER, M. (1999). Facilitatory and inhibitory effects of masked prime stimuli on motor activation and behavioral performance. Acta Psychologica, 101, 293-313. doi:10.1016/S0001-6918(99)00009-8

Eimer, M., \& SCHLAGHECKEN, F. (1998). Effects of masked stimuli on motor activation: Behavioral and electrophysiological evidence. Journal of Experimental Psychology: Human Perception \& Performance, 24, 1737-1747. doi:10.1037/0096-1523.24.6.1737

Guerin, B. (1993). Social facilitation. Cambridge: Cambridge University Press.

HARKINS, S. G. (2006). Mere effort as the mediator of the evaluationperformance relationship. Journal of Personality \& Social Psychology, 91, 436-455. doi:10.1037/0022-3514.91.3.436

Houghton, G., \& TipPeR, S. P. (1994). A model of inhibitory mechanisms in selective attention. In D. Dagenbach \& T. Carr (Eds.), Inhibitory processes in attention, memory, and language (pp. 53-112). San Diego: Academic Press.

Huguet, P., Dumas, F., \& Monteil, J.-M. (2004). Competing for a desired reward in the Stroop task: When attentional control is unconscious but effective versus conscious but ineffective. Canadian Journal of Experimental Psychology, 58, 153-167. doi:10.1037/h0087441

Huguet, P., Galvaing, M. P., Monteil, J.-M., \& Dumas, F. (1999). Social presence effects in the Stroop task: Further evidence for an attentional view of social facilitation. Journal of Personality \& Social Psychology, 77, 1011-1023. doi:10.1037/0022-3514.77.5.1011

JAMIESON, J. P., \& HaRKINS, S. G. (2007). Mere effort and stereotype threat performance effects. Journal of Personality \& Social Psychology, 93, 544-564. doi:10.1037/0022-3514.93.4.544

Klauer, K. C., Herfordt, J., \& Voss, A. (2008). Social presence effects on the Stroop task: Boundary conditions and an alternative account. Journal of Experimental Social Psychology, 44, 469-476. doi:10.1016/j.jesp.2007.02.009

MacLeod, C. M. (1991). Half a century of research on the Stroop effect: An integrative review. Psychological Bulletin, 109, 163-203. doi:10.1037/0033-2909.109.2.163

Markus, H. (1978). The effect of mere presence on social facilitation: An unobtrusive test. Journal of Experimental Social Psychology, 14 389-397. doi:10.1016/0022-1031(78)90034-3

McFall, S. R., Jamieson, J. P., \& Harkins, S. G. (2009). Testing the mere effort account of the evaluation-performance relationship. Journal of Personality \& Social Psychology, 96, 135-154. doi:10.1037/ a0012878

Melara, R. D., \& Algom, D. (2003). Driven by information: A tectonic theory of Stroop effects. Psychological Review, 110, 422-471. doi:10.1037/0033-295X.110.3.422

Muller, D., Atezeni, T., \& ButerA, F. (2004). Coaction and upward social comparison reduce the illusory conjunction effect: Support for distraction-conflict theory. Journal of Experimental Social Psychology, 40, 659-665. doi:10.1016/j.jesp.2003.12.003

RABBITT, P. [M. A.] (1980). The effects of R-S interval duration on serial choice reaction time: Preparation time or response monitoring time? Ergonomics, 23, 65-77. doi:10.1080/00140138008924719

RabBitt, P. M. A., \& MaYLoR, E. A. (1991). Investigating models of human performance. British Journal of Psychology, 82, 259-290.

RIDDERINKHOF, K. R. (2002). Activation and suppression in conflict tasks: Empirical clarification through distributional analyses. In W. Prinz \& B. Hommel (Eds.), Attention and performance XIX: Common mechanisms in perception and action (pp. 494-519). Oxford: Oxford University Press.

Ridderinkhof, K. R., Scheres, A., Oosterlaan, J., \& Sergeant, J. A. (2005). Delta plots in the study of individual differences: New tools reveal response inhibition deficits in $\mathrm{AD} / \mathrm{HD}$ that are eliminated by methylphenidate treatment. Journal of Abnormal Psychology, 114, 197-215. doi:10.1037/0021-843X.114.2.197 
Ridderinkhof, K. R., van Den Wildenberg, W. P. M., Wijnen, J., \& Burle, B. (2004). Response inhibition in conflict tasks is revealed in delta plots. In M. Posner (Ed.), Cognitive neuroscience of attention (pp. 369-377). New York: Guilford Press.

Sharma, D., \& McKenna, F. P. (2001). The role of time pressure on the emotional Stroop task. British Journal of Psychology, 92, 471-481. doi:10.1348/000712601162293

Stroop, J. (1935). Studies of interference in serial verbal reactions. Journal of Experimental Psychology, 18, 653-662. doi:10.1037/ h0054651

Van Selst, M., \& Joliceleur, P. (1994). A solution to the effect of sample size on outlier elimination. Quarterly Journal of Experimental Psychology, 47A, 631-650.

\section{NOTES}

1. Jamieson and Harkins (2007) note that it is common in the Stroop task to find differences reflected in errors when the response window is limited, but reflected in speed when it is unlimited (for relevant references, see Jamieson \& Harkins, 2007, p. 547).
2. Klauer, Herfordt, and Voss (2008) have shown that part of the instructions adopted by Huguet et al. (1999) inflated interference in the "alone" condition (see Discussion). These instructions were not used here.

3. McFall et al. (2009) found that evaluation increased errors on incongruent stimuli under a short response deadline that prevented correction of the prepotent response. Here, presence simply had no effect with short RSIs, which we believe also prevented this correction. The mere-effort account could however explain this finding if a shorter response deadline (rather than shorter preparation time) was necessary for an increased error rate to result from social presence.

4. As noted by Markus (1978, p. 391), in virtually all social facilitation studies the participant in the alone condition is not "phenomenologically alone," even when the audience and the experimenter are out of sight. This suggests that creating a true alone condition for comparison with the presence condition is in fact extremely difficult. This difficulty increases if the presence condition comes first.

(Manuscript received January 23, 2009;

revision accepted for publication October 12, 2009.) 